\title{
Small GTPases and phosphoinositides in the regulatory mechanisms of macropinosome formation and maturation
}

\author{
Youhei Egami', Tomohiko Taguchi' ${ }^{2,3}$, Masashi Maekawa ${ }^{2,4}$, Hiroyuki Arai ${ }^{2,3}$ and Nobukazu Araki ${ }^{1 *}$ \\ Department of Histology and Cell Biology, School of Medicine, Kagawa University, Miki, Japan \\ ${ }^{2}$ Department of Health Chemistry, Graduate School of Pharmaceutical Sciences, University of Tokyo, Tokyo, Japan \\ ${ }^{3}$ Pathological Cell Biology Laboratory, Graduate School of Pharmaceutical Sciences, University of Tokyo, Tokyo, Japan \\ ${ }^{4}$ Keenan Research Centre, Li Ka Shing Knowledge Institute, St. Michael's Hospital, Toronto, ON, Canada
}

\section{Edited by:}

Hiroyuki Kabayama, RIKEN Brain

Science Institute, Japan

\section{Reviewed by:}

Paul Anthony Gleeson, University of Melbourne, Australia

Robert Roger Kay, Medical Research Council, UK

\section{*Correspondence:}

Nobukazu Araki, Department of Histology and Cell Biology, School of Medicine, Kagawa University, 1750-1 Ikenobe, Miki, Kagawa 761-0793, Japan

e-mail:naraki@med.kagawa-u.ac.jp
Macropinosome formation requires the sequential activation of numerous signaling pathways that coordinate the actin-driven formation of plasma membrane protrusions (ruffles) and circular ruffles (macropinocytic cups), followed by the closure of these macropinocytic cups into macropinosomes. In the process of macropinosome formation, localized productions of phosphoinositides such as $\mathrm{PI}(4,5) \mathrm{P}_{2}$ and $\mathrm{PI}(3,4,5) \mathrm{P}_{3}$ spatiotemporally orchestrate actin polymerization and rearrangement through recruiting and activating a variety of actin-associated proteins. In addition, the sequential activation of small GTPases, which are known to be master regulators of the actin cytoskeleton, plays a pivotal role in parallel with phosphoinositides. To complete macropinosome formation, phosphoinositide breakdown and Rho GTPase deactivation must occur in appropriate timings. After the nascent macropinosomes are formed, phosphoinositides and several Rab GTPases control macropinosome maturation by regulating vesicle trafficking and membrane fusion. In this review, we summarize recent advances in our understanding of the critical functions of phosphoinositide metabolism and small GTPases in association with their downstream effectors in macropinocytosis.

Keywords: macropinocytosis, small GTPases, phosphoinositides, Rac1, optogenetics, myotubularin-related proteins

\section{INTRODUCTION}

Macropinocytosis, an actin-dependent endocytic pathway, leads to the nonselective ingestion of extracellular fluid containing nutrients, antigens, and other molecules into cells. The process was first reported as pinocytosis (drinking by cells) by Warren $\mathrm{H}$. Lewis using the earliest live-cell imaging technique, filmed time-lapse cinephotography (Lewis, 1931). Macropinocytosis, which forms large $(>0.2-5 \mu \mathrm{m})$ endocytic vacuoles called macropinosomes, was later distinguished from micropinocytosis, which is mediated by smaller vesicles $(\sim 100 \mathrm{~nm})$ such as clathrin-coated vesicles (Swanson and Watts, 1995). Compared with the mechanisms of receptor-mediated endocytosis through clathrin-coated vesicles, those of macropinocytosis have been studied less intensively in previous years. However, macropinocytosis has recently attracted increasing attention, as the important physiological and pathological implications of this process have been successively uncovered in a broad range of cell types. In macrophages and dendritic cells, exogenous proteins that are internalized through macropinocytosis are processed and presented as antigen peptides on the class II major histocompatibility complex (MHC) (Sallusto et al., 1995). In connection with pathogenesis, macrophage foam cell formation in atherosclerotic plaques can occur through the macropinocytic uptake of native low-density lipoproteins (Kruth et al., 2005). The macropinocytosis of proteins in cancer cells is a pivotal amino acid supply route for tumor growth (Commisso et al., 2013). In neuronal cells, macropinocytosis-mediated massive retrieval of the plasma membrane is an important mechanism of growth cone collapse and axon growth inhibition (Kabayama et al., 2009, 2011). Paradoxically, numerous infectious pathogens, such as bacteria, viruses, protozoa, and prions, utilize macropinocytosis for their internalization into host cells (Mercer and Helenius, 2009, 2012; Lim and Gleeson, 2011). Salmonella typhimurium induces membrane ruffling and macropinocytosis, which ultimately directs the internalization of the species into spacious phagosomes in host cells (Garcia-del Portillo and Finlay, 1994; Alpuche-Aranda et al., 1995). These organelles can be considered as similar to macropinosomes in the context of their formation mechanism. A better understanding of the molecular mechanisms underlying macropinocytosis, which serves several distinct purposes in different cell types, would provide new insights into a wide range of pathologies and human health issues.

The process of macropinocytosis begins with actin-driven plasma membrane ruffling. Although many ruffles recede soon after formation, some sheet-like ruffles turn into cupshaped circular ruffles (macropinocytic cups). Then, closure of the cup opening results in the formation of intracellular macropinosomes. Subsequently, newly formed macropinosomes undergo a process of maturation for degradation or recycling. The two key events of macropinocytosis, macropinosome formation and maturation, are mediated by the actin cytoskeleton and membrane trafficking, which are primarily controlled by 
small GTPases and phosphoinositides (Araki et al., 2000; Cardelli, 2001; Swanson, 2008; Kerr and Teasdale, 2009; Lim and Gleeson, 2011). In this review, we summarize the regulatory relevance of small GTPase and phosphoinositide metabolism to macropinocytosis and introduce our recent findings on the implications of myotubularin-related proteins (MTMRs) in phosphoinositide metabolism during macropinosome formation.

\section{SMALL GTPases}

Small GTPases of the Ras superfamily consist of several subfamilies, including the Ras, Rho, ADP ribosylation factor (Arf), and Rab GTPases, all of which are key regulators in the signaling pathways that control diverse cellular and developmental events such as differentiation, cell division, vesicle transport, nuclear assembly, and cytoskeletal organization. These GTPases are known to serve as molecular switches and regulate the process of macropinocytosis. In this section, we describe the current knowledge of the regulatory implications of each small GTPase family in macropinocytosis.

\section{Rho GTPases}

Rho family GTPases, which act as molecular switches, regulate actin cytoskeleton remodeling through cycling between an active, GTP-bound form and inactive, GDP-bound form (Ridley, 2006). Changes in the actin cytoskeleton drive many dynamic aspects of cell behavior, including morphogenesis, migration, cytokinesis, phagocytosis, and macropinocytosis. Rho GTPases encompass three groups of proteins, Rho, Rac, and $\mathrm{Cdc} 42$, which differentially regulate actin-based cell structures. Typically, Rho is involved in the formation of focal adhesion and stress fibers, Rac forms lamellipodia and/or membrane ruffles, and Cdc42 forms filopodia (Nobes and Hall, 1995; Hall, 1998; Hall and Nobes, 2000). In the process of macropinocytosis, Racl is crucial for membrane ruffling and macropinosome formation in a variety of different cell types, including dendrite cells, macrophages, fibroblasts, and epithelial cells. Furthermore, we have recently revealed that the deactivation of Rac1 following its transient activation is also required for macropinosome formation (Fujii et al., 2013). We show these data later in a separate subsection.

Cdc42 activation has also been shown in some cell types during macropinocytosis, although its necessity to macropinocytosis has not been established (Patel and Galán, 2006). In bone marrow-derived mouse dendritic cells, the microinjection of GDP-bound inactive Rac1-T17N or treatment with Clostridium difficile toxin $\mathrm{B}$, which inhibits all Rho GTPases (including Rac and Cdc42), abrogates membrane ruffling and macropinocytosis, whereas inactive Cdc42 does not affect macropinocytosis (West et al., 2000). GTP-bound Rac1 and Cdc42 activate p21-activated kinase 1 (PAK1), which is essential for ruffling and macropinosome formation (Dharmawardhane et al., 2000). PAK1 regulates actin cytoskeleton organization through WASP/WAVE-Arp $2 / 3$ activation and also phosphorylates the C-terminal-binding protein-1/Brefeldin A-ADP-ribosylated substrate (CtBP1/BARS), which is essential for the fission of macropinosomes from the plasma membrane (Liberali et al., 2008).

Although the precise roles of Rho isoforms in macropinocytosis remain unclear, the implications of some Rho isoforms in the process have been reported. Higher vertebrates possess three major Rho isoforms, RhoA, RhoB, and RhoC, which share $85 \%$ amino acid sequence identity. RhoA and RhoC GTPases share $92 \%$ amino acid sequence identity; however, they show different activity dynamics during macropinocytosis. Using FRET microscopy with a RhoC biosensor, RhoC was shown to be temporally activated at the circular ruffles prior to macropinosome closure (Zawistowski et al., 2013), while a burst of RhoA activity was observed after macropinosome closure in fibroblasts (Pertz et al., 2006; Zawistowski et al., 2013). Unlike RhoA and RhoC, RhoB localizes to intracellular endosomes and controls vesicle transport through regulating actin assembly on vesicle membranes (Fernandez-Borja et al., 2005); however, the specific contribution of RhoB to macropinosomes has not yet been determined. Additionally, RNAi knockdown and overexpression experiments have suggested that RhoG, a close homolog of Rac1, is required for the formation of dorsal membrane ruffles during growth factor-induced macropinocytosis in fibroblasts and A431 cells (Ellerbroek et al., 2004; Samson et al., 2010).

\section{Ras GTPases}

The involvement of Ras in membrane ruffling and macropinocytosis has been reported in several cell types (Bar-Sagi and Feramisco, 1986; Porat-Shliom et al., 2008; Welliver and Swanson, 2012). H-Ras localizes to membrane ruffles and forms macropinosomes in epidermal growth factor (EGF)-stimulated HeLa and COS-7 cells, and the G12V active mutant of H-Ras induces membrane ruffling and macropinocytosis (Porat-Shliom et al., 2008). In RAW macrophages, Ras activation, as measured by ratio imaging of the citrine-Ras-binding domain (RBD), is observed after circular ruffle formation and is approximately concurrent with Rab5 recruitment during macropinocytosis. The peak of Ras activity occurs slightly after cup closure (Welliver and Swanson, 2012).

\section{Arf GTPases}

The Arf family of proteins comprises another group of the Ras superfamily of small GTPases. Arf small GTPases are known to regulate membrane trafficking events, including phagocytosis and macropinocytosis, through modulating phospholipid metabolism and the actin cytoskeleton. Arf6 and Arf1, two of the best-characterized Arf proteins, have been implicated in membrane ruffling and macropinocytosis (Radhakrishna et al., 1996, 1999; Zhang et al., 1999; Schafer et al., 2000; Grimmer et al., 2002). It has been suggested that Arf6 is required for the localization of activated Racl to the plasma membrane. When Salmonella invades host cells through inducing macropinocytosis, Arf6 triggers actin assembly through recruiting ARNO, an Arf guanine nucleotide exchange factor (GEF), which activates Arf1 to enable the WAVE regulatory complex (WRC) (Humphreys et al., 2013). PI(4,5) $\mathrm{P}_{2}$ production by phosphatidylinositol 4-phosphate 5-kinase $\alpha$ (PI4P5K $\alpha)$ is stimulated through the cooperation of Arf6 and Racl in membrane ruffle formation (Honda et al., 1999). Additionally, biochemical analysis has shown that Arf6 activates phospholipase D1, which is required for macropinosome formation (Haga et al., 2009). 


\section{Rab GTPases}

Rab GTPases are key regulators of membrane trafficking in endocytic pathways (Somsel Rodman and Wandinger-Ness, 2000; Zerial and McBride, 2001). To date, more than 60 members of the Rab family have been identified in the human genome (Colicelli, 2004). Many of the Rab proteins that are localized on distinct intracellular vesicles have been reported to coordinate sequential steps of membrane transport (Schwartz et al., 2007). In the macropinocytic pathway, several Rab GTPases have been shown to be involved in macropinosome formation or subsequent macropinosome maturation. Live-cell imaging analysis and experiments with dominant-negative mutants and RNAi have revealed that Rab5 and Rab34 are involved in macropinosome formation. Porat-Shliom et al. showed that the recruitment of Rab5 to the plasma membrane overlaps with the production of phosphatidylinositol 3,4,5-triphosphate $\left(\mathrm{PI}(3,4,5) \mathrm{P}_{3}\right)$ in COS-7 cells expressing H-Ras-G12V (GTP-bound form) (Porat-Shliom et al., 2008). In MEF cells stimulated with platelet-derived growth factor (PDGF), the expression of Rab5-S34N (GDP-bound form) inhibits the induction of circular ruffles (macropinocytic cups), which are the precursors of macropinosomes (Lanzetti et al., 2004). Rab5 is recruited to macropinocytic cups together with RN-tre, a Rab5 GTPase-activating protein (GAP) and Rab5 effector, which mediates actin remodeling. Supporting the involvement of Rab5 in macropinosome formation, the expression of Rabankyrin-5, another Rab5 effector, promotes fluid-phase uptake in EGF-treated A431 cells (Schnatwinkel et al., 2004). FRET microscopy has shown that Rab5a activation follows immediately after its recruitment to nascent macropinosomes in growth factor-stimulated COS-7 cells and macrophages. Rab5a activity increases temporally in early macropinosomes and then decreases prior to its dissociation. The overexpression of activating and inhibitory proteins indicates that active Rab5a stabilizes macropinosomes (Feliciano et al., 2011).

During macropinocytosis, Rab34 is also associated with actinrich membrane ruffles and regulates macropinosome formation. In mouse embryo fibroblast $(\mathrm{C} 3 \mathrm{H} 10 \mathrm{~T} 1 / 2)$ cells, Rab34-mediated macropinocytosis requires the activity of Racl and the actin nucleation factor WAVE2 (Sun et al., 2003). However, when Coxa virus enter human intestinal epithelial Caco-2 cells through macropinocytic activity, Rab34-mediateded macropinocytosis is dependent on Ras but not Rac1 (Coyne et al., 2007).

In the process of macropinosome maturation, internalized macropinosomes migrate in a centripetal manner, contracting and rapidly acquiring late endosomal/lysosomal markers such as Rab7 (Racoosin and Swanson, 1993; Kerr et al., 2006). Although the physiological roles of each Rab protein in macropinosome maturation remain undefined, several Rab proteins are associated with macropinosomes at different points during macropinosome maturation (Figure 1). Our time-lapse observations revealed that Rab21 and Rab20 are recruited to Rab7-positive maturing macropinosomes in RAW264 macrophages (Egami and Araki, 2009, 2012). Rab21 and Rab20 are close homologs of Rab5 (Schwartz et al., 2007). These GTPases show similar but not identical spatiotemporal dynamics during macropinocytosis. Although Rab21 is largely colocalized with Rab5, the recruitment of Rab21 to the macropinosomes lags a minute behind that of Rab5 and precedes that of Rab7 (Egami and Araki, 2009). The difference between Rab5 and Rab21 is further emphasized by the recruitment of Rab21 to the macropinosomes after a decrease in $\mathrm{PI}(3,4,5) \mathrm{P}_{3}$ levels. Rab21 then dissociates from the macropinosomes prior to the accumulation of Lamp1, a late endosomal/lysosomal protein. Notably, the expression of Rab21T33N (GDP-bound form) does not inhibit macropinocytic cups or macropinosome formation, suggesting that Rab21 is unnecessary for macropinosome formation. Similarly to Rab21, Rab20 is also localized to macropinosomes. Although Rab20 is colocalized with Rab5 and Rab21 at the macropinosomal membranes, the association of Rab20 with the macropinosomes persists even after the dissociation of Rab5 and Rab21 (Egami and Araki, 2012). Intriguingly, Rab20 is colocalized both with Rab7 and Lamp1 on the macropinosomes. At present, the roles of multiple additional Rab GTPases in macropinocytosis remain under investigation.

\section{THE SIGNIFICANT ROLE OF Rac1 DEACTIVATION DURING MACROPINOSOME FORMATION}

Until recently, most studies have focused on the activation (switching on) of Rho family GTPases in membrane ruffling and macropinocytosis. However, the pivotal role of the deactivation (switching off) of these molecular switches in the completion of macropinocytosis is now becoming clear. Yoshida et al. showed the spatiotemporal activation of Racl during macropinosome formation in live macrophages using FRET image analysis, suggesting that temporal activation followed by deactivation may be important in the process (Yoshida et al., 2009).

In addition, using optogenetics to manipulate Racl activity through blue laser irradiation, we dissected the roles of Racl activation and deactivation in the process of macropinocytosis (Fujii et al., 2013). Genetically encoded photoactivatable Racl (PA-Rac1), which is a fusion of the dominant active Rac1 with the light oxygen voltage (LOV) domain and J $\alpha$-chain (Wu et al., 2009), can be locally activated in live macrophages by blue laser irradiation under a confocal microscope. When we irradiated a peripheral portion of the cell, remarkable cell surface ruffling was induced in the irradiated area. In addition, enhanced $\mathrm{PI}(4,5) \mathrm{P}_{2}$ production and actin assembly were confirmed at the region of PA-Rac1. Soon after irradiation was ceased, ruffling receded; macropinosomes were then formed from the cup or pocketshaped ruffles (Figures 2A,B). Markers of macropinosome maturation, such as $\mathrm{PI}(3) \mathrm{P}$ and Rab21, were recruited to the formed macropinosomes after the irradiation was turned off (Figure 2C). However, when we continued the irradiation, macropinosome formation was not observed; cup or pocket-like structures instead accumulated at the photoactivation region (Fujii et al., 2013; Araki et al., 2014). Thus, Racl activation alone is insufficient for closing macropinocytic cups into macropinosomes, although it efficiently forms circular ruffles. These results suggest that deactivation following the activation of Racl is crucial to the completion of macropinosome formation.

\section{PHOSPHOINOSITIDES AND LIPID-MODIFYING ENZYMES}

Phosphoinositides are produced by the phosphorylation of phosphatidylinositol (PI) on the 3, 4, and 5 positions of its inositol ring. Phosphoinositides comprise only a small fraction of cellular 


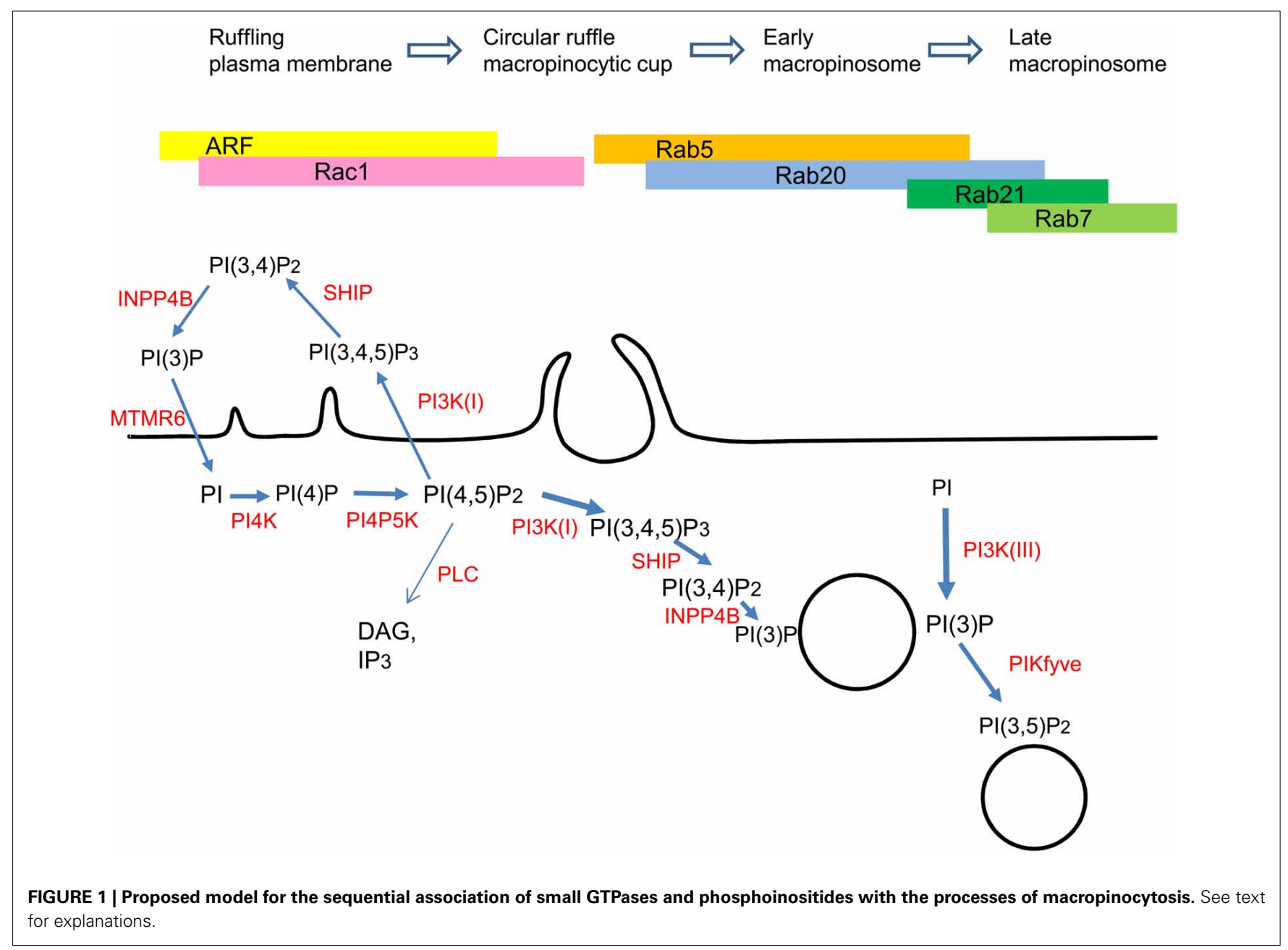

membrane phospholipids but are involved in many cellular processes, such as signal transduction and membrane dynamics (Di Paolo and De Camilli, 2006). Each phosphoinositide recruits specific proteins to the membrane domain where the phosphoinositide is enriched, then activates protein function and leads signaling cascades. The processes of macropinocytosis seem to be tightly regulated by a series of phosphoinositides with precise spatiotemporal patterns. In this section, we review the involvement of phosphoinositides in membrane ruffling, macropinocytic cup formation, macropinosome formation, and macropinosome maturation. Our recent observations of Caenorhabditis elegans, which revealed critical phosphatases that regulate phosphoinositide metabolism in membrane ruffles (Maekawa et al., 2014), are also described.

\section{PHOSPHATIDYLINOSITOL 4,5-BISPHOSPHATE $\left(\mathrm{PI}(4,5) \mathrm{P}_{2}\right)$ AND PHOSPHATIDYLINOSITOL 4-PHOSPHATE 5-KINASES (PI4P5K) IN MEMBRANE RUFFLING}

PI4P5K catalyzes the phosphorylation of phosphatidylinositol 4phosphate (PI4P) to form phosphatidylinositol 4,5-bisphosphate $\left(\mathrm{PI}(4,5) \mathrm{P}_{2}\right)$. Live-cell imaging using a fluorescent protein-fused PLC-PH domain demonstrated that the levels of $\mathrm{PI}(4,5) \mathrm{P}_{2}$ are markedly increased in membrane ruffles and macropinocytic cups (Araki et al., 2007; Welliver and Swanson, 2012). Therefore, PI4P5K must be locally activated for localized $\mathrm{PI}(4,5) \mathrm{P}_{2}$ synthesis from PI4P. In support of this finding, both Racl and ARF6, which activate PI4P5K activity (Honda et al., 1999), are known to be temporally activated at the same region (Zhang et al., 1999; Balañá et al., 2005; Yoshida et al., 2009). PI(4,5) $\mathrm{P}_{2}$ binds to activate various actin-binding proteins and initiates the actin polymerization and reorganization required for membrane ruffling, which is a prerequisite for macropinosome formation. Furthermore, $\mathrm{PI}(4,5) \mathrm{P}_{2}$ is the most common physiological substrate for phospholipase C (PLC), which produces two important messengers: diacylglycerol (DAG) and inositol trisphosphate $\left(\mathrm{IP}_{3}\right)$. DAG activates a number of protein kinase $\mathrm{C}(\mathrm{PKC})$ isoforms and causes their translocation to the plasma membrane from the cytosol. PKC activation by phorbol ester is known to enhance membrane ruffling and macropinocytosis (Swanson, 1989). The $\mathrm{PI}(4,5) \mathrm{P}_{2}$ levels in the membrane decrease upon macropinosome closure. At that time, $\mathrm{PI}(4,5) \mathrm{P}_{2}$ is hydrolyzed to $\mathrm{IP}_{3}$ and DAG by phospholipase $\mathrm{C} \gamma$ (PLC $\left.\gamma\right)$ or converted to $\mathrm{PI}(3,4,5) \mathrm{P}_{3}$ by $\mathrm{PI} 3 \mathrm{~K}$ during the process of macropinosome formation. However, PLC $\gamma$ and PI3K seem to function in distinct phases of macropinocytosis. The inhibition of PLC $\gamma$ by U-73122 perturbs macropinocytosis at the ruffle formation step, while PI3K 
A

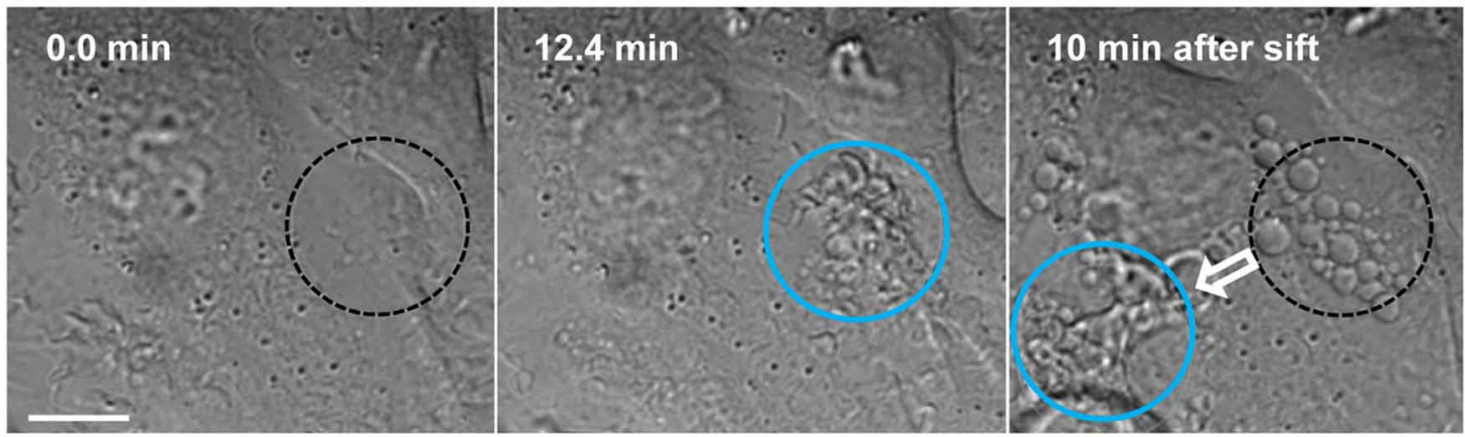

B
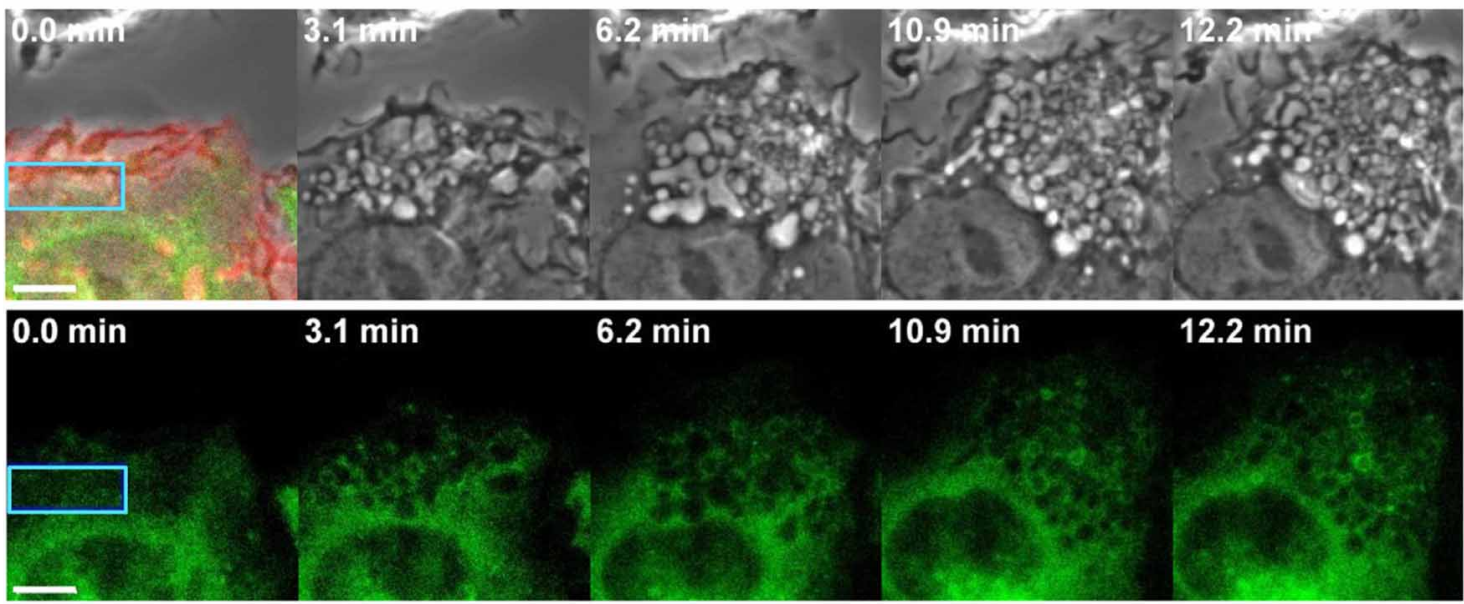

C
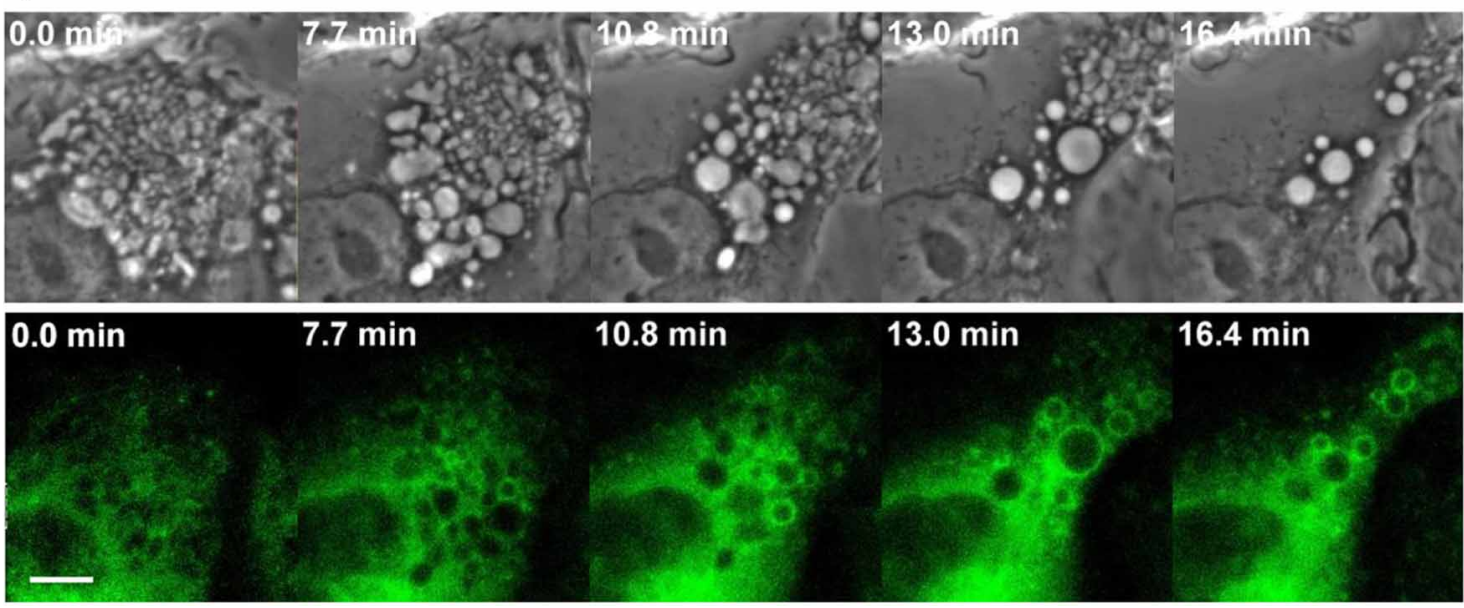

FIGURE 2 | Macropinosome formation and maturation by optogenetic control of Rac1 activation and deactivation. (A) Local and reversible control of membrane ruffling and macropinosome formation through Rac1 photoactivation and deactivation. Diffraction interference contrast images of live RAW264 macrophages expressing PA-Rac1 were acquired by confocal laser microscopy with photoactivation. Time 0 indicates the initiation of blue laser irradiation in the area enclosed by the circle. At $12.4 \mathrm{~min}$, ruffles were apparent within the irradiated region (blue circle). After $13 \mathrm{~min}$, the irradiation was shifted to a different area of the same cell. After the irradiation was ceased, the ruffles immediately receded, and spherical macropinosomes were formed in the initial irradiation area (broken-lined circle) within $10 \mathrm{~min}$. Marked ruffling was then induced in the newly irradiated area. The bar indicates $10 \mu \mathrm{m}$. (B,C) Time-lapse live-cell images of RAW264 macrophages expressing PA-Rac1 and GFP-Rab21 during photoactivation (B) and after photoactivation was ceased (C). The blue rectangular area in the cell was repeatedly irradiated using a blue laser for $12.2 \mathrm{~min}$. The top left panel shows a merged image of the phase-contrast, mCherry, and GFP fluorescence signals obtained before the photoactivation of PA-Rac1, confirming the expression of mCherry-PA-Rac1 and GFP-Rab21. The other panels show selected time-lapse phase-contrast (upper) and GFP-Rab21 images (lower; green). Time 0 indicates the initiation of photoactivation (B) or deactivation. (C) The recruitment of Rab21, a maturation marker, to the macropinosomes was found after PA-Rac1 deactivation. The bar indicates $5 \mu \mathrm{m}$ (adapted from Fujii et al., 2013). 
inhibitors block macropinocytic cup closure without affecting membrane ruffling (Araki et al., 2007). The depletion of $\mathrm{PI}(4,5) \mathrm{P}_{2}$ by PI3K and PLC may be important for the localized release of $\mathrm{PI}(4,5) \mathrm{P}_{2}$-binding proteins from the membrane (Terebiznik et al., 2002; Scott et al., 2005).

\section{PHOSPHATIDYLINOSITOL 3,4,5-TRISPHOSPHATE (PI(3,4,5)P 3 ) AND CLASS I PHOSPHATIDYLINOSITOL 3-KINASES (PI3Ks) IN MACROPINOSOME FORMATION}

The necessity of PI3K for membrane ruffling varies among different cell types and receptors. Although PDGF-induced membrane ruffling in fibroblasts is eliminated by PI3K inhibitors, membrane ruffling and circular ruffle formation in macrophages and EGF-stimulated A431 cells are resistant to these inhibitors (Araki et al., 1996, 2006, 2007). The pivotal role of class I PI3K for macropinosome closure has been well established in several cell types (Araki et al., 1996, 2006; Amyere et al., 2000).

Using live-cell imaging and scanning electron microscopy of macrophages and EGF-stimulated A431 cells, we showed that PI3K inhibitors such as LY294002 inhibit macropinosome formation but not membrane ruffling or actin assembly (Araki et al., 1996, 2006). Ratiometric imaging of fluorescent protein-fused Akt-PH or Btk-PH domains, which are fluorescent probes for $\mathrm{PI}(3,4,5) \mathrm{P}_{3}$, relative to the plasma membrane marker has demonstrated that levels of $\mathrm{PI}(3,4,5) \mathrm{P}_{3}$ are markedly increased after circular ruffle formation (Araki et al., 2007; Yoshida et al., 2009; Welliver and Swanson, 2012). The morphological features of cupshaped circular ruffles restrict the diffusion of membrane proteins and further amplify local signaling inside the cups (Welliver et al., 2011). $\mathrm{PI}(3,4,5) \mathrm{P}_{3}$ binds to the $\mathrm{PH}$ domains of several proteins, such as Akt, Btk, PDK1, and ARNO, and subsequently leads to their downstream signaling pathways. Unlike $\mathrm{PI}(4,5) \mathrm{P}_{2}$, $\mathrm{PI}(3,4,5) \mathrm{P}_{3}$ is not required for actin polymerization but instead contributes to actin depolymerization and remodeling through activating ADF/cofilin (Rupper et al., 2001; Nishita et al., 2004; Araki et al., 2007). $\mathrm{PI}(3,4,5) \mathrm{P}_{3}$ is also known to activate Rac1 through recruiting Vav, a Rac1 GEF (Patel et al., 2002).

$\mathrm{PI}(3,4,5) \mathrm{P}_{3}$ is thought to be converted into $\mathrm{PI}(3,4) \mathrm{P}_{2}$ by SHIP 5 phosphatase. Indeed, imaging of $\mathrm{PI}(3,4) \mathrm{P}_{2}$ with the TAPP domain has shown that the timing of $\mathrm{PI}(3,4) \mathrm{P}_{2}$ formation is almost simultaneous with the disappearance of $\mathrm{PI}(3,4,5) \mathrm{P}_{3}$ (Welliver and Swanson, 2012).

\section{CLASS III PHOSPHATIDYLINOSITOL 3-KINASE (PI3K) IN MACROPINOSOME MATURATION}

A few minutes after macropinosome formation, a considerable amount of $\mathrm{PI}(3) \mathrm{P}$ is found on the macropinosome membrane. Because PI(3)P production is inhibited by 3-methyladenine, a class III PI3K inhibitor, class III PI3K primarily accounts for the $\mathrm{PI}(3) \mathrm{P}$ production on macropinosomes. We have reported that the class III PI3K inhibitor prevents the recruitment of EEA1, a $\mathrm{PI}(3) \mathrm{P}$-binding membrane tethering protein, to macropinosomes and blocks the homotypic fusion of early macropinosomes (Hamasaki et al., 2004; Araki et al., 2006). Live-cell imaging of PI(3)P with the fluorescent protein-fused FYVE domain demonstrates that $\mathrm{PI}(3) \mathrm{P}$ is transiently found on the membranes of Rab5-positive early macropinosomes in macrophages
(Yoshida et al., 2009); however, in EGF-induced macropinosomes in A431 cells, $\mathrm{PI}(3) \mathrm{P}$ remains on the membranes as long as macropinosomes are present (Araki et al., 2006). Unlike macrophage macropinosomes, macropinosomes in A431 cells never mature to fuse with lysosomes. The conversion of $\mathrm{PI}(3) \mathrm{P}$ to $\mathrm{PI}(3,5) \mathrm{P}_{2}$ by PIKfyve activity may be required for further macropinosome maturation to fuse with late endosomes or lysosomes in the degradation pathway (Kerr et al., 2010). Sorting nexin 5 (SNX5), which possesses a PX domain that binds PI(3)P, is localized on EEA1-positive macropinosomes and tubular extensions from these macropinosomes (Kerr et al., 2006; Lim et al., 2008; Kerr and Teasdale, 2009). The dissociation of SNX5-positive tubules from the macropinosome is thought to promote its maturation.

\section{DEGRADATION OF PI(3,4,5) $\mathrm{P}_{\mathbf{3}}$ IN MEMBRANE RUFFLES}

In addition to the burst of $\mathrm{PI}(3,4,5) \mathrm{P}_{3}$ production inside of circular ruffles (macropinocytic cups), $\mathrm{PI}(3,4,5) \mathrm{P}_{3}$ is produced and degraded to a lesser extent in the dorsal membrane ruffles. Using fluid-phase uptake mutants of $C$. elegans, we have identified myotubularin-related proteins (MTMRs) as phosphoinositide 3-phosphatases that function at membrane ruffles (Maekawa et al., 2014). In the next two subsections, we describe the usefulness of $C$. elegans mutants in identifying important genes for macropinocytosis, as well as the sequential breakdown of $\mathrm{PI}(3,4,5) \mathrm{P}_{3} \rightarrow \mathrm{PI}(3,4) \mathrm{P}_{2} \rightarrow \mathrm{PI}(3) \mathrm{P} \rightarrow \mathrm{PI}$ in the ruffling membrane through the action of SHIP 5-phosphatase, inositol polyphosphate 4-phosphatase (INPP4), and myotubularin-related proteins (MTMRs).

\section{Fluid-phase uptake mutants in C. elegans}

C. elegans contains six scavenger-like cells, called coelomocytes, in its pseudocoelom (body cavity) (Figure 3A). Coelomocytes actively and continuously endocytose fluids and solutes to clear them from the body cavity. Considering these coelomocyte characteristics, Fares and Greenwald developed an elegant assay to monitor the endocytic activity of coelomocytes in situ (Figure 3B) (Fares and Greenwald, 2001). This assay employs a transgenic worm (arIs37) containing myo-3p::ssGFP. The transgene induces the synthesis of a secretory signal sequence-GFP chimera in the body wall muscles. GFP is secreted into the body cavity from the body wall muscles and then endocytosed by coelomocytes. GFP is thus only detected in the coelomocytes of the transgenic worms (Figures 3B,C). Through examining known viable endocytosis mutants and RNAi results for other known endocytosis genes, possible causal genes for coelomocyte uptake defective (CUP) phenotypes were determined. In these CUP mutants, GFP accumulates in the body cavity because of the defective uptake of GFP by coelomocytes (Figures 3B,C). The CUP genes identified to date are listed in Supplementary Table 1.

\section{Phosphatases that degrade 3-phosphorylated phosphoinositides in membrane ruffles}

Macropinocytosis in mammalian cells may resemble the process of fluid uptake in coelomocytes. We therefore examined if mammalian orthologs of the CUP genes are involved in macropinocytosis. We found that myotubularin-related protein 
A

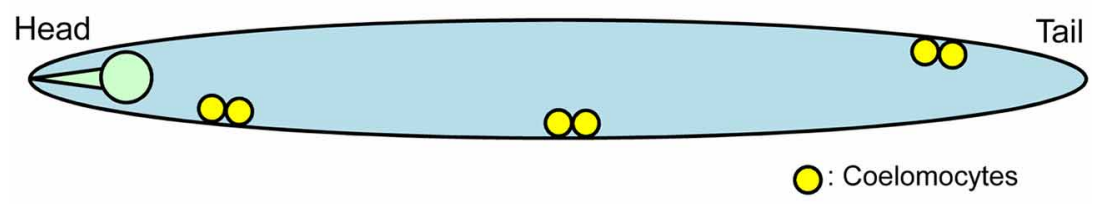

B

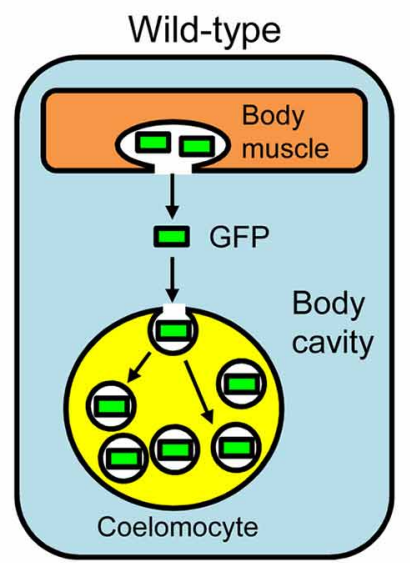

C

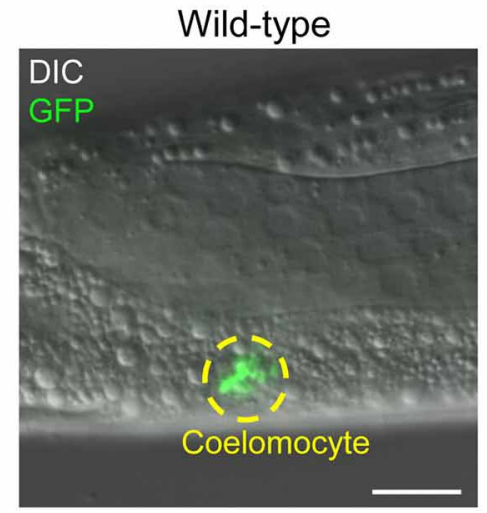

FIGURE 3 | Fluid-phase uptake in coelomocytes of Caenorhabditis elegans.(A) Caenorhabditis elegans generally possesses six coelomocytes (indicated by yellow) in its body cavity. This simple diagram of $C$. elegans is drawn from the side of the organism. Note that the other tissues of C. elegans are not shown for the sake of explanation. (B) Diagrams of the fluid-phase endocytosis assay. In wild-type worms containing myo-3p::ssGFP, GFP synthesized in the body wall muscle cells is secreted into the body cavity and taken up by coelomocytes. In wild-type worms, GFP signals are detected only in the coelomocytes. In CUP
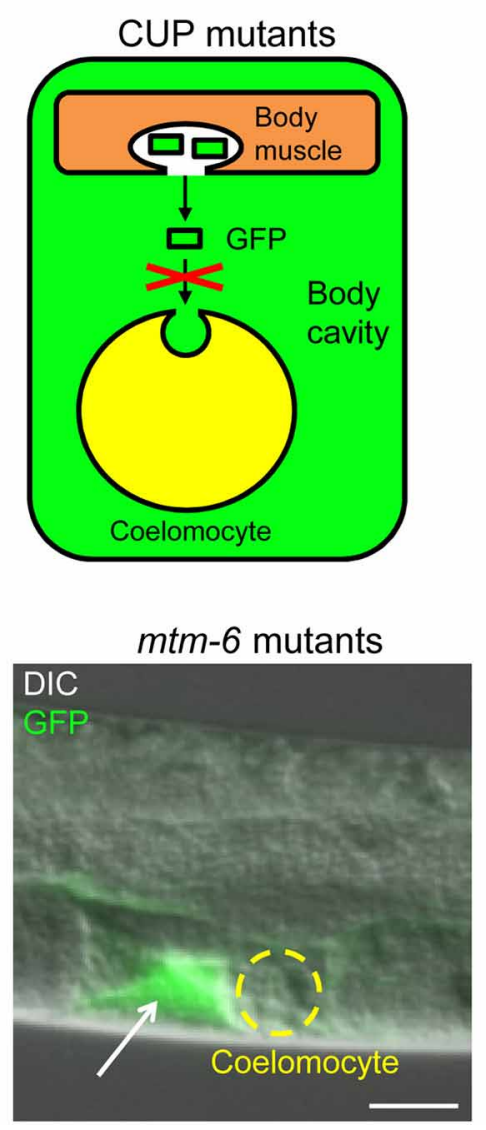

mutants containing myo-3p::ssGFP, GFP is not endocytosed by coelomocytes and is therefore accumulated in the body cavity. Note that the coelomocytes are magnified for the sake of explanation. (C) Images of wild-type worms and mtm-6(ok330)/II mutants (one of the CUP mutants; see Supplementary Table 1). All worms contain myo-3p::ssGFP. Coelomocytes in the mtm-6 mutants do not have GFP-positive vesicles, and GFP accumulates in the body cavity (indicated by a white arrow). Coelomocytes are indicated by broken yellow lines. Scale bars: $10 \mu \mathrm{m}$ (adapted from Maekawa et al., 2014).
6 (MTMR6) and myotubularin-related protein 9 (MTMR9), mammalian ortholog of $m t m-6$ and $m t m-9$, are essential for macropinocytosis in A431 cells (Maekawa et al., 2014). MTMR6 and MTMR9 belong to the myotubularin family phosphoinositide 3-phosphatases (Robinson and Dixon, 2006; Hnia et al., 2012), suggesting that the degradation of PI(3)P to PI is required for macropinosome formation. Cells depleted of MTMR6 or MTMR9 still show membrane ruffling after EGF stimulation, suggesting that MTMR6 and MTMR9 function in the steps that follow the formation of membrane ruffles (Maekawa et al., 2014).
$\mathrm{PI}(3,4,5) \mathrm{P}_{3}$ and $\mathrm{PI}(3,4) \mathrm{P}_{2}$ are transiently generated in dorsal membrane ruffles during circular ruffle formation in PDGFstimulated NIH3T3 cells (Hasegawa et al., 2011). As MTMR6 is a 3-phosphatase (Schaletzky et al., 2003), we reasoned that $\mathrm{PI}(3,4) \mathrm{P}_{2}$ is dephosphorylated by 4-phosphatase to provide the substrate $\mathrm{PI}(3) \mathrm{P}$ to MTMR6. INPP4B is a specific 4-phosphatase of $\mathrm{PI}(3,4) \mathrm{P}_{2}$ (Norris et al., 1997; Ivetac et al., 2005; Gewinner et al., 2009). Using RNAi knockdown experiments, we found that INPP4B is essential for macropinocytosis in EGF-stimulated A431 cells (Maekawa et al., 2014). Similarly to the knockdowns of MTMR6 and MTMR9, the knockdown of INPP4B did not inhibit 
EGF-induced membrane ruffling. Therefore, INPP4B likely also functions at the steps after the formation of membrane ruffles.

In light of a report that SHIP2, a 5-phosphatase of $\mathrm{PI}(3,4,5) \mathrm{P}_{3}$, is involved in PDGF-induced circular ruffle formation in NIH3T3 cells after PDGF (Hasegawa et al., 2011), these results suggest that $\mathrm{PI}(3,4,5) \mathrm{P}_{3}$ is sequentially converted to $\mathrm{PI}$ in membrane ruffles by specific PI phosphatases and that this process is indispensable for circular ruffle/macropinocytic cup formation. Live cell imaging of EGF-stimulated A431 cells expressing fluorescent Akt-PH or TAPP1-PH domains has consistently detected significant levels of $\mathrm{PI}(3,4,5)_{3}$ and $\mathrm{PI}(3,4) \mathrm{P}_{2}$ at the membrane of EGF-stimulated ruffling (Maekawa et al., 2014), although these increased levels are not as high as those detected on the membrane inside of macropinocytic cups after circular ruffle formation (Welliver and Swanson, 2012).

When monitoring PI(3)P using a GFP-fused FYVE domain, $\mathrm{PI}(3) \mathrm{P}$ is faintly observed in the ruffling membrane. However, in MTMR6-depeleted A431 cells with RNAi, significant levels of $\mathrm{PI}(3) \mathrm{P}$ are detected in the membrane of EGF-induced dorsal ruffling. Double knockdown of MTMR6 and SHIP2 or of MTMR6 and INPP4B prevents the emergence of PI(3)P after EGF stimulation, indicating that the $\mathrm{PI}(3) \mathrm{P}$ in the membrane ruffles is produced through the sequential breakdown of $\mathrm{PI}(3,4,5) \mathrm{P}_{3}$ and $\mathrm{PI}(3,4) \mathrm{P}_{2}$ by SHIP2 and INPP4B. The level of $\mathrm{PI}(3) \mathrm{P}$ in the membrane ruffle is much lower than that in the membranes of early macropinosomes, which is produced through the phosphorylation of PI by class III PI3K. However, the transient $\mathrm{PI}(3) \mathrm{P}$ production in the membrane ruffle may be required for the activation of $\mathrm{KCa} 3.1$, a $\mathrm{Ca}^{2+}$-activated $\mathrm{K}^{+}$channel (Srivastava et al., 2005). Actually, KCa3.1 localizes to membrane ruffles and is essential for macropinosome formation (Maekawa et al., 2014).

\section{MACROPINOCYTOSIS IN DICTYOSTELIUM DISCOIDEUM}

Dictyostelium discoideum is a genetically and biochemically tractable soil ameba. In $D$. discoideum, active macropinocytosis constitutively occurs for the uptake of nutrients from the environment and can be experimentally detected through the observation of macropinosomes labeled by fluorophore-conjugated dextran, a fluid-phase marker, added to the medium. Therefore, the molecular mechanisms of macropinocytosis in D. discoideum have been elucidated, as have those of phagocytosis and chemotaxis, using gene disruption and protein expression (Maniak, 2001). Similarly to that in mammalian cells, macropinocytosis in D. discoideum is actin-dependent (Hacker et al., 1997). Null mutants of coronin, an actin-binding protein, and Daip1, an actin-interacting protein, present defective macropinocytosis (Maniak et al., 1995; Konzok et al., 1999). Rac1 GTPase also regulates macropinocytosis in D. discoideum (Dumontier et al., 2000). Another study using null mutants and an inhibitor has indicated that class I PI3K and its downstream effector, protein kinase $\mathrm{B}(\mathrm{PKB} / \mathrm{Akt})$, regulate macropinocytosis in this species (Rupper et al., 2001). More recently, Hoeller et al. (2013) revealed two distinct functions for class I PI3K isoforms in macropinocytosis using systematic genetic ablation. PI3K1 and 2 produce patches of $\mathrm{PI}(3,4,5) \mathrm{P}_{3}$ associated with actin-dependent ruffle formation, and PI3K4 is required for the conversion of ruffles into intracellular macropinosomes. Furthermore, these authors identified two specific Ras proteins, RasG and RasS, that interact with PI3K1/2 and PI3K4 (Hoeller et al., 2013).

Through expressing probes for phosphoinositides, the dynamics of 3-phosphoinositides can be visualized during macropinocytosis in D. discoideum (Dormann et al., 2004; Hoeller et al., 2013; Veltman et al., 2014). PI(3,4,5) $\mathrm{P}_{3}$ transiently increases at the macropinocytic cups, followed by rapid $\mathrm{PI}(3,4) \mathrm{P}_{2}$ generation at the periphery of the macropinosomes upon internalization, suggesting that $\mathrm{PI}(3,4,5) \mathrm{P}_{3}$ is degraded to $\mathrm{PI}(3,4) \mathrm{P}_{2}$ through macropinosome maturation (Dormann et al., 2004). Similar phosphoinositide kinetics are observed during macropinocytosis in mammalian macrophages (Welliver and Swanson, 2012). Thus, this free-living ameba shares many features of macropinocytosis with mammalian phagocytes such as macrophages (Cardelli, 2001). Although careful attention should be given to the differences in the molecular mechanisms of macropinocytosis between $D$. discoideum and mammalian cells, information from this species is generally helpful for the analysis of mammalian macropinocytosis.

\section{CONCLUDING REMARKS}

Our current knowledge of the macropinocytic pathway in mammals has been primarily derived from pharmacological and gene overexpression approaches. However, due to the off-target effects or incomplete inhibition, it is occasionally difficult to identify a single key gene for a certain process of macropinocytosis. In this respect, genetic studies of $C$. elegans and $D$. discoideum are useful for identifying crucial regulators of macropinocytosis, although careful attention must be given to the differences in the molecular mechanisms among different organisms. Additionally, the application of newly developed genome editing systems such as CRISPR/Cas9 may shed light on this endocytic mechanism in mammalian cells. To better understand the dynamic mechanisms coordinating macropinosome formation and maturation in both space and time, both the genetic identification of key molecules and live cell imaging are crucial. Combining morphological studies with FRET imaging, super-resolution microscopy, and/or optogenetics will be quite valuable for dissecting the dynamic molecular mechanisms of this process.

Although still incompletely understood, the molecular mechanism that controls macropinocytosis has been greatly elucidated in recent years. It is now apparent that large numbers of signaling and mechanistic molecules spatiotemporally regulate the coordinated processes of macropinosome formation and maturation. The complex interplay of small GTPase molecular switches and phosphoinositides orchestrates the highly sophisticated processes of macropinocytosis (Figure 1). However, the regulation of macropinocytosis may partly be dependent on cell type. Macropinocytosis is constitutive in antigen-presenting cells such as macrophages and dendritic cells (Steinman and Swanson, 1995; Swanson and Watts, 1995), but it can also be rapidly induced by growth factors in other cell types, such as epithelial cells and fibroblasts. Macropinosomes usually mature and merge with the lysosomal degradative pathway. However, macropinosomes in certain cell types, such as A431 cells, do not deliver their fluid contents to the degradation pathway and are instead extracellularly regurgitated by recycling pathways 
(Hewlett, 1994; Hamasaki et al., 2004). Although the biological significance of this backflow is unknown, it is of special interest for elucidating the differences in the molecular mechanisms underlying the distinct emergence and fate of macropinosomes in different cell types. If we can determine these differences using molecular and signaling comparisons of the two distinct macropinocytic pathways, we can manipulate macropinocytosis through activating or inhibiting the key signals. Several pathogenic microorganisms that enter thorough macropinocytosis interfere with macropinosome maturation to survive inside of the cell. The manipulation of macropinocytosis would therefore also be to our advantage in developing therapeutic strategies for human diseases involve macropinocytic activity.

\section{ACKNOWLEDGMENTS}

This work was funded by Japan Society for the Promotion of Science (JSPS) KAKENHI to Nobukazu Araki and Youhei Egami. Additional financial supports provided by JSPS, Ministry of Agriculture, Forestry and Fisheries of Japan, and Japan Science and Technology Agency to Hiroyuki Arai are also acknowledged. The authors would like to thank Dr. Makoto Fujii and Dr. Katsuhisa Kawai for their scientific contributions to our recent studies.

\section{SUPPLEMENTARY MATERIAL}

The Supplementary Material for this article can be found online at: http://www.frontiersin.org/journal/10.3389/fphys. 2014.00374/abstract

\section{REFERENCES}

Alpuche-Aranda, C. M., Berthiaume, E. P., Mock, B., Swanson, J. A., and Miller, S. I. (1995). Spacious phagosome formation within mouse macrophages correlates with Salmonella serotype pathogenicity and host susceptibility. Infect. Immun. 63, 4456-4462.

Amyere, M., Payrastre, B., Krause, U., Van Der Smissen, P., Veithen, A., and Courtoy, P. J. (2000). Constitutive macropinocytosis in oncogene-transformed fibroblasts depends on sequential permanent activation of phosphoinositide 3-kinase and phospholipase C. Mol. Biol. Cell 11, 3453-3467. doi: $10.1091 / \mathrm{mbc} .11 .10 .3453$

Araki, N., Egami, Y., Watanabe, Y., and Hatae, T. (2007). Phosphoinositide metabolism during membrane ruffling and macropinosome formation in EGF-stimulated A431 cells. Exp. Cell Res. 313, 1496-1507. doi: 10.1016/j.yexcr.2007.02.012

Araki, N., Hamasaki, M., Egami, Y., and Hatae, T. (2006). Effect of 3-methyladenine on the fusion process of macropinosomes in EGF-stimulated A431 Cells. Cell Struct. Funct. 31, 145-157. doi: 10.1247/csf.06029

Araki, N., Hatae, T., Yamada, T., and Hirohashi, S. (2000). Actinin-4 is preferentially involved in circular ruffling and macropinocytosis in mouse macrophages: analysis by fluorescence ratio imaging. J. Cell Sci. 113, 3329-3340.

Araki, N., Ikeda, Y., Kato, T., Kawai, K., Egami, Y., Miyake, K., et al. (2014). Development of an automated fluorescence microscopy system for photomanipulation of genetically encoded photoactivatable proteins (optogenetics) in live cells. Microscopy 63, 255-260. doi: 10.1093/jmicro/dfu003

Araki, N., Johnson, M. T., and Swanson, J. A. (1996). A role for phosphoinositide 3-kinase in the completion of macropinocytosis and phagocytosis by macrophages. J. Cell Biol. 135, 1249-1260. doi: 10.1083/jcb.135.5.1249

Balañá, M. E., Niedergang, F., Subtil, A., Alcover, A., Chavrier, P., and DautryVarsat, A. (2005). ARF6 GTPase controls bacterial invasion by actin remodelling. J. Cell Sci. 118, 2201-2210. doi: 10.1242/jcs.02351

Bar-Sagi, D., and Feramisco, J. R. (1986). Induction of membrane ruffling and fluid-phase pinocytosis in quiescent fibroblasts by ras proteins. Science 233, 1061-1068. doi: 10.1126/science.3090687
Cardelli, J. (2001). Phagocytosis and macropinocytosis in Dictyostelium: phosphoinositide-based processes, biochemically distinct. Traffic 2, 311-320. doi: 10.1034/j.1600-0854.2001.002005311.x

Colicelli, J. (2004). Human RAS superfamily proteins and related GTPases. Sci. STKE 2004:RE13. doi: 10.1126/stke.2502004re13

Commisso, C., Davidson, S. M., Soydaner-Azeloglu, R. G., Parker, S. J., Kamphorst, J. J., Hackett, S., et al. (2013). Macropinocytosis of protein is an amino acid supply route in Ras-transformed cells. Nature 497, 633-637. doi: $10.1038 /$ nature 12138

Coyne, C. B., Shen, L., Turner, J. R., and Bergelson, J. M. (2007). Coxsackievirus entry across epithelial tight junctions requires occludin and the small GTPases Rab34 and Rab5. Cell Host Microbe 2, 181-192. doi: 10.1016/j.chom.2007. 07.003

Dharmawardhane, S., Schurmann, A., Sells, M. A., Chernoff, J., Schmid, S. L., and Bokoch, G. M. (2000). Regulation of macropinocytosis by p21-activated kinase-1. Mol. Biol. Cell 11, 3341-3352. doi: 10.1091/mbc.11.10.3341

Di Paolo, G., and De Camilli, P. (2006). Phosphoinositides in cell regulation and membrane dynamics. Nature 443, 651-657. doi: 10.1038/nature05185

Dormann, D., Weijer, G., Dowler, S., and Weijer, C. J. (2004). In vivo analysis of 3-phosphoinositide dynamics during Dictyostelium phagocytosis and chemotaxis. J. Cell Sci. 117, 6497-6509. doi: 10.1242/jcs.01579

Dumontier, M., Höcht, P., Mintert, U., and Faix, J. (2000). Rac1 GTPases control filopodia formation, cell motility, endocytosis, cytokinesis and development in Dictyostelium. J. Cell Sci. 113, 2253-2265.

Egami, Y., and Araki, N. (2009). Dynamic changes in the spatiotemporal localization of Rab21 in live RAW264 cells during macropinocytosis. PLoS ONE 4:e6689. doi: 10.1371/journal.pone.0006689

Egami, Y., and Araki, N. (2012). Spatiotemporal localization of Rab20 in live RAW264 macrophages during macropinocytosis. Acta Histochem. Cytochem. 45, 317-323. doi: 10.1267/ahc.12014

Ellerbroek, S. M., Wennerberg, K., Arthur, W. T., Dunty, J. M., Bowman, D. R., DeMali, K. A., et al. (2004). SGEF, a RhoG guanine nucleotide exchange factor that stimulates macropinocytosis. Mol. Biol. Cell 15, 3309-3319. doi: 10.1091/mbc.E04-02-0146

Fares, H., and Greenwald, I. (2001). Genetic analysis of endocytosis in Caenorhabditis elegans: coelomocyte uptake defective mutants. Genetics 159, 133-145.

Feliciano, W. D., Yoshida, S., Straight, S. W., and Swanson, J. A. (2011). Coordination of the Rab5 cycle on macropinosomes. Traffic 12, 1911-1922. doi: 10.1111/j.1600-0854.2011.01280.x

Fernandez-Borja, M., Janssen, L., Verwoerd, D., Hordijk, P., and Neefjes, J. (2005). RhoB regulates endosome transport by promoting actin assembly on endosomal membranes through Dial. J. Cell Sci. 118, 2661-2670. doi: 10.1242/jcs. 02384

Fujii, M., Kawai, K., Egami, Y., and Araki, N. (2013). Dissecting the roles of Racl activation and deactivation in macropinocytosis using microscopic photomanipulation. Sci. Rep. 3:2385. doi: 10.1038/srep02385

Garcia-del Portillo, F., and Finlay, B. B. (1994). Salmonella invasion of nonphagocytic cells induces formation of macropinosomes in the host cell. Infect. Immun. 62, 4641-4645.

Gewinner, C., Wang, Z. C., Richardson, A., Teruya-Feldstein, J., Etemadmoghadam, D., Bowtell, D., et al. (2009). Evidence that inositol polyphosphate 4phosphatase type II is a tumor suppressor that inhibits PI3K signaling. Cancer Cell 16, 115-125. doi: 10.1016/j.ccr.2009.06.006

Grimmer, S., van Deurs, B., and Sandvig, K. (2002). Membrane ruffling and macropinocytosis in A431 cells require cholesterol. J. Cell Sci. 115, 2953-2962.

Hacker, U., Albrecht, R., and Maniak, M. (1997). Fluid-phase uptake by macropinocytosis in Dictyostelium. J. Cell Sci. 110, 105-112.

Haga, Y., Miwa, N., Jahangeer, S., Okada, T., and Nakamura, S. (2009). CtBP1/BARS is an activator of phospholipase D1 necessary for agonist-induced macropinocytosis. EMBO J. 28, 1197-1207. doi: 10.1038/emboj.2009.78

Hall, A. (1998). Rho GTPases and the actin cytoskeleton. Science 279, 509-514. doi: $10.1126 /$ science. 279.5350 .509

Hall, A., and Nobes, C. D. (2000). Rho GTPases: molecular switches that control the organization and dynamics of the actin cytoskeleton. Philos. Trans. R. Soc. Lond. B Biol. Sci. 355, 965-970. doi: 10.1098/rstb.2000.0632

Hamasaki, M., Araki, N., and Hatae, T. (2004). Association of early endosomal autoantigen 1 with macropinocytosis in EGF-stimulated A431 cells. Anat. Rec. A Discov. Mol. Cell. Evol. Biol. 277, 298-306. doi: 10.1002/ar.a.20027 
Hasegawa, J., Tokuda, E., Tenno, T., Tsujita, K., Sawai, H., Hiroaki, H., et al. (2011). SH3YL1 regulates dorsal ruffle formation by a novel phosphoinositide-binding domain. J. Cell Biol. 193, 901-916. doi: 10.1083/jcb.201012161

Hewlett, L. J. (1994). The coated pit and macropinocytic pathways serve distinct endosome populations. J. Cell Biol. 124, 689-703. doi: 10.1083/jcb.124.5.689

Hnia, K., Vaccari, I., Bolino, A., and Laporte, J. (2012). Myotubularin phosphoinositide phosphatases: cellular functions and disease pathophysiology. Trends Mol. Med. 18, 317-327. doi: 10.1016/j.molmed.2012.04.004

Hoeller, O., Bolourani, P., Clark, J., Stephens, L. R., Hawkins, P. T., Weiner, O. D., et al. (2013). Two distinct functions for PI3-kinases in macropinocytosis. J. Cell Sci. 126, 4296-4307. doi: 10.1242/jcs. 134015

Honda, A., Nogami, M., Yokozeki, T., Yamazaki, M., Nakamura, H., Watanabe, H., et al. (1999). Phosphatidylinositol 4-phosphate 5-kinase alpha is a downstream effector of the small G protein ARF6 in membrane ruffle formation. Cell 99, 521-532. doi: 10.1016/S0092-8674(00)81540-8

Humphreys, D., Davidson, A. C., Hume, P. J., Makin, L. E., and Koronakis, V. (2013). Arf6 coordinates actin assembly through the WAVE complex, a mechanism usurped by Salmonella to invade host cells. Proc. Natl. Acad. Sci. U.S.A. 110, 16880-16885. doi: 10.1073/pnas.1311680110

Ivetac, I., Munday, A. D., Kisseleva, M. V., Zhang, X.-M., Luff, S., Tiganis, T., et al. (2005). The type Ialpha inositol polyphosphate 4-phosphatase generates and terminates phosphoinositide 3-kinase signals on endosomes and the plasma membrane. Mol. Biol. Cell 16, 2218-2233. doi: 10.1091/mbc.E04-09-0799

Kabayama, H., Nakamura, T., Takeuchi, M., Iwasaki, H., Taniguchi, M., Tokushige, N., et al. (2009). $\mathrm{Ca}^{2+}$ induces macropinocytosis via F-actin depolymerization during growth cone collapse. Mol. Cell. Neurosci. 40, 27-38. doi: 10.1016/j.mcn.2008.08.009

Kabayama, H., Takeuchi, M., Taniguchi, M., Tokushige, N., Kozaki, S., Mizutani, A., et al. (2011). Syntaxin 1B suppresses macropinocytosis and semaphorin 3A-induced growth cone collapse. J. Neurosci. 31, 7357-7364. doi: 10.1523/JNEUROSCI.2718-10.2011

Kerr, M. C., Lindsay, M. R., Luetterforst, R., Hamilton, N., Simpson, F., Parton, R. G., et al. (2006). Visualisation of macropinosome maturation by the recruitment of sorting nexins. J. Cell Sci. 119, 3967-3980. doi: 10.1242/jcs.03167

Kerr, M. C., and Teasdale, R. D. (2009). Defining macropinocytosis. Traffic 10, 364-371. doi: 10.1111/j.1600-0854.2009.00878.x

Kerr, M. C., Wang, J. T. H., Castro, N. A., Hamilton, N. A., Town, L., Brown, D. L., et al. (2010). Inhibition of the PtdIns(5) kinase PIKfyve disrupts intracellular replication of Salmonella. EMBO J. 29, 1331-1347. doi: 10.1038/emboj. 2010.28

Konzok, A., Weber, I., Simmeth, E., Hacker, U., Maniak, M., and MüllerTaubenberger, A. (1999). DAip1, a Dictyostelium homologue of the yeast actin-interacting protein 1, is involved in endocytosis, cytokinesis, and motility. J. Cell Biol. 146, 453-464. doi: 10.1083/jcb.146.2.453

Kruth, H. S., Jones, N. L., Huang, W., Zhao, B., Ishii, I., Chang, J., et al. (2005). Macropinocytosis is the endocytic pathway that mediates macrophage foam cell formation with native low density lipoprotein. J. Biol. Chem. 280, 2352-2360. doi: 10.1074/jbc.M407167200

Lanzetti, L., Palamidessi, A., Areces, L., Scita, G., and Di Fiore, P. P. (2004). Rab5 is a signalling GTPase involved in actin remodelling by receptor tyrosine kinases. Nature 429, 309-314. doi: 10.1038/nature02542

Lewis, W. H. (1931). Pinocytosis. Bull. Johns Hopkins Hosp. 49, 17-26.

Liberali, P., Kakkonen, E., Turacchio, G., Valente, C., Spaar, A., Perinetti, G., et al. (2008). The closure of Pak1-dependent macropinosomes requires the phosphorylation of CtBP1/BARS. EMBO J. 27, 970-981. doi: 10.1038/emboj.2008.59

Lim, J. P., and Gleeson, P. A. (2011). Macropinocytosis: an endocytic pathway for internalising large gulps. Immunol. Cell Biol. 89, 836-843. doi: 10.1038/icb.2011.20

Lim, J. P., Wang, J. T. H., Kerr, M. C., Teasdale, R. D., and Gleeson, P. A. (2008). A role for SNX5 in the regulation of macropinocytosis. BMC Cell Biol. 9:58. doi: 10.1186/1471-2121-9-58

Maekawa, M., Terasaka, S., Mochizuki, Y., Kawai, K., Ikeda, Y., Araki, N., et al. (2014). Sequential breakdown of 3-phosphorylated phosphoinositides is essential for the completion of macropinocytosis. Proc. Natl. Acad. Sci. U.S.A. 111, E978-E987. doi: 10.1073/pnas.1311029111

Maniak, M. (2001). Fluid-phase uptake and transit in axenic Dictyostelium cells. Biochim. Biophys. Acta 1525, 197-204. doi: 10.1016/S0304-4165(01)00105-2

Maniak, M., Rauchenberger, R., Albrecht, R., Murphy, J., and Gerisch, G. (1995). Coronin involved in phagocytosis: dynamics of particle-induced relocalization visualized by a green fluorescent protein tag. Cell 83, 915-924. doi: 10.1016/0092-8674(95)90207-4

Mercer, J., and Helenius, A. (2009). Virus entry by macropinocytosis. Nat. Cell Biol. 11, 510-520. doi: 10.1038/ncb0509-510

Mercer, J., and Helenius, A. (2012). Gulping rather than sipping: macropinocytosis as a way of virus entry. Curr. Opin. Microbiol. 15, 490-499. doi: 10.1016/j.mib.2012.05.016

Nishita, M., Wang, Y., Tomizawa, C., Suzuki, A., Niwa, R., Uemura, T., et al. (2004). Phosphoinositide 3-kinase-mediated activation of cofilin phosphatase Slingshot and its role for insulin-induced membrane protrusion. J. Biol. Chem. 279, 7193-7198. doi: 10.1074/jbc.M312591200

Nobes, C. D., and Hall, A. (1995). Rho, Rac, and Cdc42 GTPases regulate the assembly of multimolecular focal complexes associated with actin stress fibers, lamellipodia, and filopodia. Cell 81, 53-62. doi: 10.1016/0092-8674(95)90370-4

Norris, F. A., Atkins, R. C., and Majerus, P. W. (1997). The cDNA cloning and characterization of inositol polyphosphate 4-phosphatase type II. Evidence for conserved alternative splicing in the 4-phosphatase family. J. Biol. Chem. 272, 23859-23864. doi: 10.1074/jbc.272.38.23859

Patel, J. C., and Galán, J. E. (2006). Differential activation and function of Rho GTPases during Salmonella-host cell interactions. J. Cell Biol. 175, 453-463. doi: $10.1083 /$ jcb. 200605144

Patel, J. C., Hall, A., and Caron, E. (2002). Vav regulates activation of Rac but not Cdc42 during FcgammaR-mediated phagocytosis. Mol. Biol. Cell 13, 1215-1226. doi: 10.1091/mbc.02-01-0002

Pertz, O., Hodgson, L., Klemke, R. L., and Hahn, K. M. (2006). Spatiotemporal dynamics of RhoA activity in migrating cells. Nature 440, 1069-1072. doi: 10.1038/nature04665

Porat-Shliom, N., Kloog, Y., and Donaldson, J. G. (2008). A unique platform for H-Ras signaling involving clathrin-independent endocytosis. Mol. Biol. Cell 19, 765-775. doi: 10.1091/mbc.E07-08-0841

Racoosin, E. L., and Swanson, J. A. (1993). Macropinosome maturation and fusion with tubular lysosomes in macrophages. J. Cell Biol. 121, 1011-1020. doi: 10.1083/jcb.121.5.1011

Radhakrishna, H., Al-Awar, O., Khachikian, Z., and Donaldson, J. G. (1999). ARF6 requirement for Rac ruffling suggests a role for membrane trafficking in cortical actin rearrangements. J. Cell Sci. 112, 855-866.

Radhakrishna, H., Klausner, R. D., and Donaldson, J. G. (1996). Aluminum fluoride stimulates surface protrusions in cells overexpressing the ARF6 GTPase. J. Cell Biol. 134, 935-947. doi: 10.1083/jcb.134.4.935

Ridley, A. J. (2006). Rho GTPases and actin dynamics in membrane protrusions and vesicle trafficking. Trends Cell Biol. 16, 522-529. doi: 10.1016/j.tcb.2006.08.006

Robinson, F. L., and Dixon, J. E. (2006). Myotubularin phosphatases: policing 3phosphoinositides. Trends Cell Biol. 16, 403-412. doi: 10.1016/j.tcb.2006.06.001

Rupper, A., Lee, K., Knecht, D., and Cardelli, J. (2001). Sequential activities of phosphoinositide 3-kinase, PKB/Aakt, and Rab7 during macropinosome formation in Dictyostelium. Mol. Biol. Cell 12, 2813-2824. doi: 10.1091/mbc.12. 9.2813

Sallusto, F., Cella, M., Danieli, C., and Lanzavecchia, A. (1995). Dendritic cells use macropinocytosis and the mannose receptor to concentrate macromolecules in the major histocompatibility complex class II compartment: downregulation by cytokines and bacterial products. J. Exp. Med. 182, 389-400. doi: 10.1084/jem.182.2.389

Samson, T., Welch, C., Monaghan-Benson, E., Hahn, K. M., and Burridge, K. (2010). Endogenous RhoG is rapidly activated after epidermal growth factor stimulation through multiple guanine-nucleotide exchange factors. Mol. Biol. Cell 21, 1629-1642. doi: 10.1091/mbc.E09-09-0809

Schafer, D. A., D'Souza-Schorey, C., and Cooper, J. A. (2000). Actin assembly at membranes controlled by ARF6. Traffic 1, 896-907. doi: 10.1034/j.16000854.2000.011108.x

Schaletzky, J., Dove, S. K., Short, B., Lorenzo, O., Clague, M. J., and Barr, F. A. (2003). Phosphatidylinositol-5-phosphate activation and conserved substrate specificity of the myotubularin phosphatidylinositol 3-phosphatases. Curr. Biol. 13, 504-509. doi: 10.1016/S0960-9822(03)00132-5

Schnatwinkel, C., Christoforidis, S., Lindsay, M. R., Uttenweiler-Joseph, S., Wilm, M., Parton, R. G., et al. (2004). The Rab5 effector Rabankyrin-5 regulates and coordinates different endocytic mechanisms. PLoS Biol. 2:E261. doi: 10.1371/journal.pbio.0020261

Schwartz, S. L., Cao, C., Pylypenko, O., Rak, A., and Wandinger-Ness, A. (2007). Rab GTPases at a glance. J. Cell Sci. 120, 3905-3910. doi: 10.1242/jcs.015909 
Scott, C. C., Dobson, W., Botelho, R. J., Coady-Osberg, N., Chavrier, P., Knecht, D. A., et al. (2005). Phosphatidylinositol-4,5-bisphosphate hydrolysis directs actin remodeling during phagocytosis. J. Cell Biol. 169, 139-149. doi: $10.1083 /$ jcb.200412162

Somsel Rodman, J., and Wandinger-Ness, A. (2000). Rab GTPases coordinate endocytosis. J. Cell Sci. 113, 183-192.

Srivastava, S., Li, Z., Lin, L., Liu, G., Ko, K., Coetzee, W. A., et al. (2005). The phosphatidylinositol 3-phosphate phosphatase myotubularin- related protein 6 (MTMR6) is a negative regulator of the $\mathrm{Ca}^{2+}$-activated $\mathrm{K}^{+}$channel KCa3.1. Mol. Cell. Biol. 25, 3630-3638. doi: 10.1128/MCB.25.9.3630-3638.2005

Steinman, R. M., and Swanson, J. (1995). The endocytic activity of dendritic cells. J. Exp. Med. 182, 283-288. doi: 10.1084/jem.182.2.283

Sun, P., Yamamoto, H., Suetsugu, S., Miki, H., Takenawa, T., and Endo, T. (2003). Small GTPase Rah/Rab34 is associated with membrane ruffles and macropinosomes and promotes macropinosome formation. J. Biol. Chem. 278, 4063-4071. doi: 10.1074/jbc.M208699200

Swanson, J. A. (1989). Phorbol esters stimulate macropinocytosis and solute flow through macrophages. J. Cell Sci. 94, 135-142.

Swanson, J. A. (2008). Shaping cups into phagosomes and macropinosomes. Nat. Rev. Mol. Cell Biol. 9, 639-649. doi: 10.1038/nrm2447

Swanson, J. A., and Watts, C. (1995). Macropinocytosis. Trends Cell Biol. 5, 424-428. doi: 10.1016/S0962-8924(00)89101-1

Terebiznik, M. R., Vieira, O. V., Marcus, S. L., Slade, A., Yip, C. M., Trimble, W. S., et al. (2002). Elimination of host cell PtdIns $(4,5) \mathrm{P}_{2}$ by bacterial SigD promotes membrane fission during invasion by Salmonella. Nat. Cell Biol. 4, 766-773. doi: $10.1038 /$ ncb854

Veltman, D. M., Lemieux, M. G., Knecht, D. A., and Insall, R. H. (2014). PIP3dependent macropinocytosis is incompatible with chemotaxis. J. Cell Biol. 204, 497-505. doi: 10.1083/jcb.201309081

Welliver, T. P., Chang, S. L., Linderman, J. J., and Swanson, J. A. (2011). Ruffles limit diffusion in the plasma membrane during macropinosome formation. J. Cell Sci. 124, 4106-4114. doi: 10.1242/jcs.091538

Welliver, T. P., and Swanson, J. A. (2012). A growth factor signaling cascade confined to circular ruffles in macrophages. Biol. Open 1, 754-760. doi: 10.1242/bio. 20121784

West, M. A., Prescott, A. R., Eskelinen, E.-L., Ridley, A. J., and Watts, C. (2000). $\mathrm{Rac}$ is required for constitutive macropinocytosis by dendritic cells but does not control its downregulation. Curr. Biol. 10, 839-848. doi: 10.1016/S09609822(00)00595-9

Wu, Y. I., Frey, D., Lungu, O. I., Jaehrig, A., Schlichting, I., Kuhlman, B., et al. (2009). A genetically encoded photoactivatable Rac controls the motility of living cells. Nature 461, 104-108. doi: 10.1038/nature 08241

Yoshida, S., Hoppe, A. D., Araki, N., and Swanson, J. A. (2009). Sequential signaling in plasma-membrane domains during macropinosome formation in macrophages. J. Cell Sci. 122, 3250-3261. doi: 10.1242/jcs.053207

Zawistowski, J. S., Sabouri-Ghomi, M., Danuser, G., Hahn, K. M., and Hodgson, L. (2013). A RhoC biosensor reveals differences in the activation kinetics of RhoA and RhoC in migrating cells. PLoS ONE 8:e79877. doi: 10.1371/journal.pone. 0079877

Zerial, M., and McBride, H. (2001). Rab proteins as membrane organizers. Nat. Rev. Mol. Cell Biol. 2, 107-117. doi: 10.1038/35052055

Zhang, Q., Calafat, J., Janssen, H., and Greenberg, S. (1999). ARF6 is required for growth factor- and rac-mediated membrane ruffling in macrophages at a stage distal to rac membrane targeting. Mol. Cell. Biol. 19, 8158-8168.

Conflict of Interest Statement: The authors declare that the research was conducted in the absence of any commercial or financial relationships that could be construed as a potential conflict of interest.

Received: 17 July 2014; accepted: 10 September 2014; published online: 30 September 2014.

Citation: Egami Y, Taguchi T, Maekawa M, Arai H and Araki N (2014) Small GTPases and phosphoinositides in the regulatory mechanisms of macropinosome formation and maturation. Front. Physiol. 5:374. doi: 10.3389/fphys.2014.00374

This article was submitted to Membrane Physiology and Membrane Biophysics, a section of the journal Frontiers in Physiology.

Copyright (c) 2014 Egami, Taguchi, Maekawa, Arai and Araki. This is an openaccess article distributed under the terms of the Creative Commons Attribution License (CC BY). The use, distribution or reproduction in other forums is permitted, provided the original author(s) or licensor are credited and that the original publication in this journal is cited, in accordance with accepted academic practice. No use, distribution or reproduction is permitted which does not comply with these terms. 